\title{
Prolonged haemodialysis increases the risk of the impairment of global longitudinal strain in patient after kidney transplantation and a preserved ejection fraction of left ventricle
}

\section{Marta Obremska ( $\nabla$ mobremska@gmail.com )}

Uniwersytet Medyczny im Piastow Slaskich we Wroclawiu https://orcid.org/0000-0003-1937-1891

\section{Anna Goździk}

Uniwersytet Medyczny im Piastow Slaskich we Wroclawiu

Maciej Szymczak

Uniwersytet Medyczny im Piastow Slaskich we Wroclawiu

Dorota Zysko

Uniwersytet Medyczny im Piastow Slaskich we Wroclawiu

Maria Boratyńska

Karkonoska Panstwowa Szkola Wyzsza w Jeleniej Gorze

Krzysztof Letachowicz

Uniwersytet Medyczny im Piastow Slaskich we Wroclawiu

Marcin Madziarski

Uniwersytet Medyczny im Piastow Slaskich we Wroclawiu

Magdalena Krajewska

Uniwersytet Medyczny im Piastow Slaskich we Wroclawiu

Research article

Keywords: global longitudinal strain, kidney transplantation, haemodialysis, preserved left ventricle ejection fraction.

Posted Date: November 6th, 2019

DOI: https://doi.org/10.21203/rs.2.14684/v2

License: (9) (1) This work is licensed under a Creative Commons Attribution 4.0 International License.

Read Full License 


\section{Abstract}

Background. Patients with chronic kidney disease, including these after kidney transplantation (KTx), have higher cardiovascular mortality. Global longitudinal strain (GLS) detects subtle changes in the left ventricle (LV) and is more sensitive predictor of cardiovascular mortality than the LV ejection fraction (LVEF).The aim of this study was to assess the prevalence of impaired GLS among kidney transplant recipients with preserved LVEF. We also aimed to identify possible clinical factors responsible for GLS impairment.

Methods. A total of 79 patients following KTx with preserved LVEF and no history of cardiac disease were evaluated. We assessed echocardiogram parameters with the calculation of GLS, laboratory parameters, presence of diabetes, hypertension, duration of haemodialysis (HD) and time after KTx. An impaired GLS value was set on $\geq-18 \%$. The multivariate stepwise logistic regression analysis were used to identify the factors related to impaired GLS.

Results. Among 79 (42 females, mean age 60.3) kidney transplant recipients with preserved LVEF, 39\% had impaired GLS. ROC analysis revealed that the cut-off point for the prediction GLS $\geq-18 \%$ by HD duration is more than 28 months ( $\mathrm{AUC} 0.67(95 \% \mathrm{Cl} 0.545-0.79 ; \mathrm{P}=0.007)$. In multivariate stepwise logistic regression analysis for variables related to impaired GLS duration of HD more than 28month is associated with GLS $\geq-18$ ( OR 4.06; 95Cl (1.45-11.34) $\mathrm{P}=0.008)$.

Conclusions. In our study group, a total of $39 \%$ of KTx patients with preserved LVEF had impaired GLS. The risk factor of GLS impairment was a prolonged duration of HD (>28months) prior to the transplantation procedure.

\section{Background}

Cardiovascular disease is the major threat for survival of patients with chronic kidney disease (CKD) including those after kidney transplantation (KTx). The risk of cardiovascular disease rises by $15 \%$ for every increasing stage of CKD [1]. The CKD from stage 3 to the end-stage renal disease (ESRD) is associated with volume and pressure overload as well as with renal specific risk factors for cardiomyopathy leading to left ventricular hypertrophy (LVH), reduced capillary density, and increased myocardial fibrosis.

During dialysis treatment abnormal myocardial remodelling and LVH progress due to additional factors such as bone mineral disorders, inflammation, oxidative stress, arterio-venous fistula, and many others. The LVH is observed at about $75 \%$ of adult patients and $69 \%$ of paediatric patients after the initiation of dialysis, and among almost all patients after 5 years on dialysis therapy $[2,3,4]$. LVH and increased myocardial fibrosis are causes of coronary disease, heart failure, arrhythmia and sudden cardiac death.

Successful KTx improves long-term survival in comparison to dialysis patients. However, the risk of cardiovascular disease is still three- to five-fold higher in comparison to the age-matched general 
population [5].

$\mathrm{KTx}$ reduces some of the factors of cardiomyopathy, such as volume and pressure overload, and uremia; but immunosuppressive treatment based on calcineurin inhibitors and steroids contributes to hypertension and metabolic disorders. Moreover, most of the kidney transplant recipients have renal impairment and are in stage 3 CKD according to KDIGO (Kidney Disease: Improving Global Outcomes).

CKD patients, including kidney transplant patients, with preserved left ventricle ejection fraction (LVEF), and with no symptoms of cardiovascular disease have subclinical left ventricle (LV) systolic dysfunction, which is not detected in the standard echocardiography. Detection of a subtle damage of LV systolic contractility is possible by speckle-tracking echocardiography (STE) using global longitudinal strain (GLS).

GLS is a percentage change in length of myocardial segment in systole and is presented as negative value. The prognostic value of impaired GLS of LV is well documented among patients with various cardiovascular diseases. In recent years some studies have confirmed impaired GLS as a predictor of allcause and cardiovascular mortality among patients with CKD $[6,7]$. So far, there have been no studies on clinical factors affecting GLS impairment in kidney transplant recipients.

The aim of the study was to assess the prevalence of impaired GLS among kidney transplant recipients with preserved LVEF and to identify the clinical factors for reduced GLS in these patients.

\section{Methods}

\section{Patient population}

We performed a retrospective analysis of patients after a successful KTx. The patients were derived from the Transplant Outpatient Clinic, which come from the Department of Nephrology and Transplantation Medicine of the University Hospital, where about 100 transplants are performed annually in the last 10 years. The documentation of all patients of the Transplantation Outpatient Clinic who were examined between 2015 and 2018 was analyzed. From this study group, patients who underwent echocardiographic examination with GLS assessment in the central echo room of the hospital were selected. The indication for the echocardiographic examination was usually physical dyspnea reported by the patient.Inclusion criteria were ejection fraction above $50 \%$, presence of sinus rhythm and good visualization enabling GLS assessment. Exclusion criteria were severe or moderate valvular pathology, presence of atrial fibrillation or coronary artery disease in medical history. Finally, 79 patients were included in the study. Patients' demographic and clinical data were extracted from the patient's electronic medical records and then verified with paper charts. Demographic and clinical data included: age, gender, body mass index (BMI), presence of diabetes (DM), hypertension (HTN), duration of haemodialysis (HD) before and after KTx. In addition, the type of immunosuppressive regimen based on the mammalian target of rapamycin inhibitors (mTORi) or calcineurin inhibitors (CNI) has been reported. Each patient had their blood pressure measured prior to echocardiography. Laboratory variables included both serum lipid 
concentration and assessment of renal graft function (e.g. serum creatinine, estimated glomerular filtration rate (eGFR), and uric acid). The eGFR was calculated based on the Modification of Diet in Renal Disease formula. In the case of GFR, a mean GFR was used for the analysis, which was obtained from the previous 3 tests conducted every 3 months in the last six months before the echocardiographic examination. The last collected variables refer to the date of the echocardiographic examination.

\section{Echocardiography}

Transthoracic echocardiography examination was performed for each patient for clinical indications using a high-resolution ultrasound machine (GE Vivid E 9, Horten, Norway) in accordance with the European Society for Echocardiography recommendations. All measurements and STE analysis were performed by an experienced echocardiographer (O.M). The linear measurements of the LV, such as enddiastolic diameter (LVEDd), intraventricular end-diastolic diameter (IVDd), posterior end-diastolic wall thickness diameter (PWTd), were obtained by using the M-mode ultrasound imaging. Based on these measurements the LV mass (LVM) was calculated and then indexed to the body surface area (BSA).

The upper limit of the LV mass index (LVMI) was set on $115 \mathrm{~g} / \mathrm{m}^{2}$ for men and $95 \mathrm{~g} / \mathrm{m}^{2}$ for women. Severe $\mathrm{LVH}$ was considered as $L V M I \geq 149 / \mathrm{m}^{2}$ and $\geq 122 / \mathrm{m}^{2}$ for men and women, respectively. The relative wall thickness (RWT) was calculated for classified LV geometry, using the following formula: $R W T=$ $(2 P W T d) / L V E D d)$. The concentric LVH was diagnosed in the presence of the following factors: increased LVMI, normal LV size and RWT $>0.42$, whereas the eccentric LVH was diagnosed in the presence of an increased LVMI, increased LV size, and RWT $\leq 0.42$. The LVEF and was measured using the modified Simson's rule. A similar formula was used for measured left atrium volume which was then indexed by BSA. The Doppler echocardiography was used to evaluate the diastolic function of LV such as the early diastolic mitral inflow (E) and late diastolic mitral inflow (A) in order to derive E/A ratio and deceleration time. Doppler tissue imaging was performed in a four-chamber view to measure lateral and septal velocities of the mitral annulus, such as the peak early diastolic filling velocity (e) and the peak late diastolic filling velocity (a). Therefore, the ratio of $E$ to average e (lateral+septal/2) was evaluated. GLS was measured using a two-dimensional STE and was then provided by the software as the average peak systolic longitudinal strain value of the three apical views and expressed as a negative value. The normal value of GLS was considered below $-18 \%$. [8,9]. This value as a cut-off point was also adopted in studies of subclinical systolic dysfunction in patients with diabetes [10].Basically, the more negative the GLS value, the better cardiac function. Additionally, our study population was divided into two groups according to LV GLS $<-18 \%$ (with normal GLS) and $\geq-18 \%$ (with impaired GLS).

\section{Statistical analysis}

Continues variables were presented as mean and standard deviation or medians and interquartile ranges depending on their distribution and compared with Student's T-test or Mann Whitney U test as appropriate. The Shapiro-Wilk test was used to assess the variables' distribution. The discrete variables were presented as nouns and percentages and were compared with the Chi-square test with Yates 
correction when indicated. The duration of HD before KTX and time elapsed after KTx were analysed both as continuous variables and were dichotomised using ROC curve analysis for prediction of GLS $\geq 18 \%$.

The multivariate stepwise logistic regression analysis was performed to find independent variables related to $G L S \geq-18 \%$. The factors which in univariate analysis differ between groups with $P$ less than 0.15 and variables of clinical significance were used as independent variables. A $P$-value of less than 0.05 was regarded as statistically significant.

\section{Results}

A total of 79 (53\% females) kidney transplant recipients at mean (SD) age 60.3 (11.5) were included in the study. Among the reasons of KTx were: glomerulonephritis in $28(35,4)$ patients, polycystic kidney disease in 16 (20.3\%), HTN in 12 (15.2\%), nephrolithiasis in 2 (2.5\%), carcinoma in 1 (1.3\%), kidney defects in $6(7.6 \%)$, DM in $3(3.8 \%)$, interstitial nephritis in $1(1.3 \%)$, and the cause was unknown in 10 $(12,6 \%)$ patients. The average (SD) duration of renal replacement therapy was 144 (80.6) months and included the time of HD before KTx, and time from KTx up to the day the echocardiography examination was performed. The mean (SD) time after KTx was 120.2 (71.5) months, while the mean (SD) time of HD before KTx was 23.4 (23.4) months,. Ten patients did not undergo HD before KTx.

Patients were divided into two groups depending on the GLS value. Group 1 consisted of 31 patients with GLS $\geq-18 \%$, whereas Group 2 consisted of 48 patients with GLS $<-18 \%$. The demographic and clinical characteristic of the two groups is shown in Table 1. Both groups were similar in terms of age, women's presence and BMI.

The frequency of administration of the mTORi-based immunosuppressive regimen was similar in two groups. Only 2 patients from Group 1 and 4 patients from Group 2 received mTORi de novo after KTx as a protocol drug. While 10 patients from Group1 and 16 from Group2 were converted from immunosuppressive regimen based on $\mathrm{CNI}$ to mTORi due to neoplastic disease or signs $\mathrm{CNI}$ nephrotoxicity treatment..

There were no significant differences between groups in the incidence of DM, HTN. The laboratory results were similar in both groups and are presented in Table 2.Althought all patients had preserved LVEF, Group 1 had significant higher LVEF. Echocardiographic parameters in study groups are shown in Table 3

LVH was observed in 65 (82\%) patients. The incidence of severe LVH as well as concentric and eccentric LVH were similar in both groups. There was no significant difference between groups in Doppler parameters assessing the diastolic function of LV. The mean (SD) value of average GLS for all patients was $-19.3 \%(-3,2 \%)$, however, Group 1 had significantly higher mean value of mean GLS $(P<0.005)$. ROC analysis revealed that the cut-off point for the prediction GLS $\geq-18 \%$ by HD duration is more than 28 months. The area under the ROC curve was $0.67(95 \% \mathrm{Cl} 0.545-0.79 ; P=0.007)$ (Figure 1$) \cdot$ ROC analysis with time after KTx did not determinate the cut-off point related to GLS. $\geq-18 \%$. The area under ROC curve for time after KTx was $0.58(95 \% \mathrm{Cl} 0.45-0.71 ; P=0.23)$ (Figure 2). Ten patients who did not undergo 
HD before KTx had a normal value of GLS. The mean (SD) time after KTx in these patients was 95.3 (51.8) months, whereas the mean (SD) eGFR was $41.3(9.1) \mathrm{mL} / \mathrm{min} / 1.73 \mathrm{~m}^{2}$. In multivariate stepwise logistic regression analysis for variables related to impaired GLS only duration of HD is associated with GLS $\geq-18 \%$ : as a continuous variable OR (unit change):1.03;95\% $\mathrm{Cl}(1.003-1.06), P=0.025$. A similar analysis using dichotomized HD revealed that HD longer than 28 months significantly increases the risk for GLS impairment: OR 4.06; 95Cl (1.45-11.34) $P=0.008$.

\section{Discussion}

This study demonstrates that impaired GLS occurs in nearly $40 \%$ of KTx patients with preserved LVEF and that GLS impairment is related to the duration of HD before KTX

In our study, the majority of KTx patients had severe LVH. The LVH did not correlate with the impaired GLS. LVMI was comparable in patients with or without impaired GLS. Although patients with damaged GLS had a statistically lower EF, both groups had preserved EF, which is considered a good prognosis.

Impaired GLS in CKD patients is an indicator of decreased LV contractility probably due to LV remodelling with increased accumulation of extracellular matrix and myocardial fibrosis. Recently, Sandal et al. found that congestive heart failure, but not coronary artery disease, is the most common cause of cardiovascular events following KTx [11]. That finding confirms earlier outcomes of studies based on the United States Renal Data System conducted on thousands of patient [12]. The consensus was that the KTx is a state of "accelerated heart failure" rather than "accelerated atherosclerosis".

The advantages of LV GLS over LVEF in the assessment of LV systolic function have been demonstrated in several studies in the general population as well as cardiac disease and in CKD patients. The previously reported prevalence of impairment of LV GLS in CKD patients with preserved LVEF ranged from 17 to $60 \%$. The differences can be attributed to the selection of CKD patients for the studies (with various stages of CKD from 1 to 5 , including patients with cardiac morbidity, e.g. history of myocardial infarction, CABG, atrial fibrillation, heart failure), and various cut-off values of GLS (-18 to $-15 \%)$. Panoulas et al. in their study carried out on 106 CKD patients with LVEF $\geq 55 \%$ and no history of cardiovascular disease or its symptoms, defined the GLS impairment as values greater than $-16 \%$ [13]. The incidence of impaired GLS was dependent on the CKD stage and was only $3.4 \%$ in stage 1 or 2 , $39.5 \%$ in stage 3 , and $25.6 \%$ in stage 4 and 5 . While the greatest LVMI was observed in patient $4-5$ stage. In patients with impaired GLS, the authors observed an increased rate of adverse cardiovascular events (including general mortality, coronary artery disease, and length of hospitalization for heart failure) during follow-up of $30.7 \pm 11.7$ months. They also suggested that impaired GLS may be a result of microvascular ischemia and myocardial fibrosis as the studied group did not have an evident disease in epicardial arteries, which had been confirmed by elective angiography. Hensen et al. showed LV systolic dysfunction defined by LV GLS $\leq 15.2 \%$. in $32 \%$ of predialysis and dialysis patients with $L V$ ejection fraction $\geq 50 \%$. The cut-off value for impaired GLS was obtained from 2 standard deviations below the mean LV GLS value derived from healthy controls [14]. Ravera et al. observed GLS impairment (defining the cut-off of 
GLS impairment value on less than -18\%) in 55\% of CKD patients in stage $2-4$ and in $60 \%$ of dialysed patients [15].In kidney transplant patients, according to various studies the value of GLS was -17 to $19 \%$, because CKD patients with lower GLS were less frequently transplanted. In Hensen study, only $8 \%$ patients with GLS < 10.6\% were transplanted, while 32\% with GLS 10.7-15.1\%; 49\% with GLS 15.2$17.8 \%$, and $47 \%$ with GLS > 17.9\% [16].

In prospective observational studies of patients with hypertension or diabetes, the cut-off points for GLS described as LV systolic dysfunction and associated with adverse cardiovascular events were similar. The Lee et al study of 94 patients with hypertension showed that GLS> $17.6 \%$ was associated with cardiovascular adverse events[17] In Holland et al. study, with asymptomatic patients with diabetes and preserved LVEF, the all-cause mortality was related with GLS above 18.9\% [18]

Based on oncological studies with cardiotoxicity of chemotherapy, it was established that not a specific threshold but decrease in GLS by more than 15\% from the baseline statement is associated with significant LV systolic dysfunction, which requires cardioprotective treatment [19].

Many previous studies have shown that KTx has a beneficial effect on LV structure and function compared to dialysis. The studies based on a series of conventional echocardiograms showed an improvement in LVEF after KTx. Ravinder et al. showed improvement not only in LVEF but also in functional status of heart failure and increased survival rate after KTx [20]. Paoletti et al. in their study also proved that the LVH regression following KTx was a predictor of a better long-term clinical outcome [21]. However, the LVM regression has not been confirmed by CMR what may raise doubts about the reversibility of LVH in transplant patients [22].

It has not been established whether GLS ameliorates following KTx. There are only a few studies presenting GLS in small groups of patients before and after KTx. A retrospective study of Hewing et al. showed improvement of LV global longitudinal peak systolic strain (GLPS) after KTx [23]. They evaluated a group of 31 CKD patients at mean age 44 years before and 13-32 (mean 19) months after successful KTx and found significant improvement in LV systolic function assessed by GLS $(-18.4 \% \pm 2.8 \%$ vs $19.4 \% \pm 2.3 \%)$. Concomitantly, reduction of LV end-diastolic septal and posterior wall thickness and LVMI were observed. In that study 8 patients (25\%) had preemptive KTx.

The analysis of LV systolic function assessed by speckle-tracking echocardiography was reported in pediatric patients with stage 3-5 CKD, during dialysis treatment, and after KTx. The study revealed higher impairment of GLS in patients on dialysis than in stage 3-5 CKD and in patients after KTx. One year after KTx, children presented impairment of GLS similar to stage 3-5 CKD, suggesting improvement of LV function. However, it cannot be determined whether impaired systolic contractility assessed by GLS did improve after KTx (in comparison to dialysis children) because $42 \%$ of patients (18 out of 42 ) had preemptive KTx [24].

Contrary to these studies, Gong et al. presented greater impairment of GLS in kidney transplant patients. They conducted a prospective study in 39 patients (mean age 47 years) who underwent KTx. LV GLS was 
assessed by cardiovascular magnetic resonance imaging (CMRI) at baseline and at 12 months after KTx. Kidney transplant patients had decline in GLS over 12 months $(-15.9 \% \pm 3 \%$ vs $-14.9 \% \pm 3 \%)$ posttransplantation, although LVEF and LV volumes improved, pointing to a reduction of LVH [25].

Our study indicates that the duration of HD longer than 28months leads to 4 -fold increase of impaired GLS. This results may suggest that the structural abnormalities of myocardium progress on HD and fail to subside after KTx. Our study for the first time showed the relation of subclinical LV systolic dysfunction with a duration of HD before KTx. Relationship between LVH and duration of HD was reported. Foley et al. showed that after 18 months of dialysis $62 \%$ of the patients had increased LV mass volume index and $49 \%$ of them developed overt LV failure [26].

Many studies showed, that long time on HD before transplantation is associated with decreased patient and kidney allograft survival. The Meier-Krieshe study, including 73,103 patients with the United States Renal Transplant Scientific Registry, evidenced that longer time on HD prior to KTx compared to preemptive KTx was a significant risk factor for the death of a patient with a functioning graft $(p<0.001)$. Increase in mortality risk after KTx was $41 \%$ for patients on HD for $24-36$ months and $72 \%$ for patients dialyzed over 48 months relative to preemptive transplantation [27].

Recently, Jay et al. reassessed preemptive KTx using data of 141,254 transplant recipients from United Network of Organ Sharing, who were transplanted between 2003 and 2012. Their retrospective study confirmed that preemptive KTx, as well as a short time of dialysis $<1$ year prior to transplantation in recipients of living kidney donor were associated with higher 5-year patient survival. During the last 2 decades, the percentage of patients with preemptive KTx raised from $10.9 \%$ to $17 \%$ [28].

During HD the patients are exposed to multiple factors such pressure and volume overload, activation of renin-angiotensin system chronic inflammation, hyperhomocysteinemia, advanced glycosylation end products, anaemia, endothelial dysfunction, oxidative stress, arteriovenous access. The cumulative effect of these factors leads to cardiac and vascular damage. Dialysis treatment compared to preemptive KTx was associated with increased stiffness and reduced vascular compliance. Yet, the important role of FGF23 should emphasized, which serum level increase over 400 times in hemodialysed patients. FGF23 levels are associated with LVH, and myocardial fibrosis, and increased cardiovascular mortality [29].

Ten patients in our study with preemptive KTx had normal GLS, although they were mean 95 months after transplantation and had lower eGFR $\left(41.3 \mathrm{~mL} / \mathrm{min} / 1.73 \mathrm{~m}^{2}\right)$ This outcome emphasises the most harmful role of HD on systolic function on LV.

\section{Study Limitation}

The first limitation is the lack of comparison with echocardiograms before and after hemodialysis. Such a comparison would provide more information about the course and possible reversibility of GLS damage. The second limitation is the evaluation of only longitudinal strain, which only evaluates 
subendocardial myofibres without assessment of radial and circumferential strain, that evaluate midwall myofibres.

\section{Conclusions}

In total, $39 \%$ of kidney transplant patients with preserved LVEF and no history of ischemic heart diseases had reduced LV contractility defined as impaired GLS.

The main risk factor of GLS impairment was a long period of HD (>28) prior to the transplantation procedure.

\section{List Of Abbreviations}

Alate diastolic mitral inflow

Apeak late diastolic filling velocity

BMlbody mass index

BSAbody surface area

CKDchronic kidney diseases

DMdiabetes mellitus

Eearly diastolic mitral inflow

eearly diastolic filling velocity

eGRFestimated glomerular filtration rate

GLSglobal longitudinal strain

HDhaemodialysis

HTNhypertension

IVDd intraventricular end-diastolic diameter

LAVI indexed left atrium volume

LVleft ventricle

LVEDdleft ventricle end-diastolic diameter

LVEFleft ventricle ejection fraction 
LVHleft ventricle hypertrophy

LVMleft ventricle mass

LVMlleft ventricle mass index

KTxkindey transplantation

PWTdposterior end-diastolic wall thickness diameter

ROC Receiver Operating Characteristics

RWTrelative wall thickness

STEspeckle-tracking echocardiography

\section{Declarations}

Ethics approval and consent to participate.

The study is retrospective and has been approved by the Ethics Committee of Wroclaw Medical University -The statement No 530/2019

The consent for publication

Not applicable

Availability of data and materials

The datasets used and analysed during the current study are available from the corresponding author on reasonable request.

Competing interests

The authors declare that they have no competing interests.

Funding.

The study was conducted by employees of the Wroclaw Medical University as part of scientific activity.

Authors' contributions

OM performed the echocardiography examinations and was a major contributor in writing the manuscript. OM, GA, SM contributed to the design of the research. SM BM, LK KM analyzed and interpreted the patients' data regarding haemodialysis and transplantation. All the authors were involved 
in data collection. OM, ZD performed statistical analysis. All authors read and approved the final version of the manuscript.

Acknowledgements

Not applicable

\section{References}

1. Whaley-Connell AT, Sowers JR, Stevens LA et al. Program Investigators. CKD in the United States: Kidney Early Evaluation Program (KEEP) and National Health and Nutrition Examination Survey (NHANES) 1999-2004. Am J Kidney Dis.2008;51:S13-20.

2. Foley RN, Parfrey PS, Harnett JD et al. Clinical and echocardiographic disease in patients starting end-stage renal disease therapy. Kidney Int. 1995;47:186-92.

3. Foley RN, Parfrey PS, Kent GM et al. Serial change in echocardiographic parameters and cardiac failure in end-stage renal disease. J Am Soc Nephrol. 2000;11:912-16.

4. Zoccali C, Benedetto FA, Mallamaci F et al. Left ventricular mass monitoring in the follow-up of dialysis patients: prognostic value of left ventricular hypertrophy progression. Kidney Int. 2004;65:1492-98.

5. Bottomley MJ, Harden PN. Update on the long-term complications of renal transplantation. Br Med Bull. 2013;106:117-34.

6. Krishnasamy R, Isbel NM, Hawley CM et al. Left Ventricular Global Longitudinal Strain (GLS) Is a Superior Predictor of All-Cause and Cardiovascular Mortality When Compared to Ejection Fraction in Advanced Chronic Kidney Disease. PLoS One. 2015;10(5):e0127044.

7. Fujikura K, Peltzer B, Tiwari $\mathrm{N}$ et al. Reduced global longitudinal strain is associated with increased risk of cardiovascular events or death after kidney transplant. Int J Cardiol. 2018;272: 323-28.

8. Potter E, Marwick TH. Assessment of Left Ventricular Function by Echocardiography: The Case for Routinely Adding Global Longitudinal Strain to Ejection Fraction. JACC Cardiovasc Imaging. 2018;11:260-74.

9. Marwick TH, Leano RL, Brown J, Sun JP, Hoffmann R, Lysyansky P, et al. Myocardial strain measurement with 2-dimensional speckle-tracking echocardiography: definition of normal range. JACC Cardiovasc Imaging. 2009;2(1):80-4.

10. Mochizuki Y, Tanaka H, Matsumono K, Sano H, Toki H Shimoura H et al. Clinical features of subclinical left ventricular systolic dysfunction in patients with diabetes mellitus. Cardiovasc Diabetol 2015;14;37.

11. Sandal S, Bae S, McAdams-DeMarco M et al. Induction immunosuppression agents as risk factors for incident cardiovascular events and mortality after kidney transplantation. Am J Transplant. 2019;19:1150-59. 
12. Foley RN, Collins AJ. The USRDS: what you need to know about what it can and can't tell us about ESRD. Clin J Am Soc Nephrol. 2013;8:845-51.

13. Panoulas VF, Sulemane $S$, Konstantinou K et al. Early detection of subclinical left ventricular myocardial dysfunction in patients with chronic kidney disease. Eur Heart J Cardiovasc Imaging. 2015;16:539-48.

14. Hensen LCR, Goossens K, Delgado Vet al. Prevalence of left ventricular systolic dysfunction in predialysis and dialysis patients with preserved left ventricular ejection fraction. Eur $\mathrm{J}$ Heart Fail. 2018;20: 560-68.

15. Ravera M, Rosa GM, Fontanive $P$ et al. Left Ventricular Global Longitudinal Strain among Patients with Chronic Kidney Disease and End-Stage Renal Disease and Renal Transplant Recipients. Cardiorenal Med. 2019;9:61-68.

16. Hensen LCR, Goossens K,Delgado V,Rotmans JI,Jukema JW,Bax JJ.Prognostic Implications of Left Ventricular Global Longitudinal Strain in Predialysis and Dialysis Patients. Am J Cardiol. 2017;120:500-4.

17. Lee WH, Liu YW, Yang LT, Tsai WC. Prognostic value of longitudinal strain of subepicardial myocardium in patients with hypertension. J Hypertens. 2016;34:1195-200

18. Holland DJ, Marwick TH, Haluska BA, Leano R, Hordern MD, Hare JL et al. Subclinical LV dysfunction and 10-year outcomes in type 2 diabetes mellitus. Heart. 2015;101:1061-6.

19. Zamorano JL, Lancellotti P, Rodriguez Munoz D, Aboyans V Asteggiano R, Galderisi M. et al. ESC Scientific Document Group 2016 ESC Position Paper on cancer treatments and cardiovascular toxicity developed under the auspies of the ESC Committee for Practice Guidelines: The Task Force for cancer treatments and cardiovascular toxicity of the European Society of Cardiology (ESC) Eur Heart J 2016;37:2768-2801.

20. Ravinder K, Wali RK, Wang GS, Gottlieb SS, Bellumkonda L, Hansalia R, Ramos E, Drachenberg C, Papadimitriou J, Brisco MA, Blahut S, Fink JC, Fisher ML, Bartlett ST, Weir MR. Effect of kidney transplantation on left ventricular systolic dysfunction and congestive heart failure in patients with end-stage renal disease. J Am Coll Cardiol. 2005;45:1051-60.

21. Paoletti E, Bellino D, Signori A et al. Regression of asymptomatic cardiomyopathy and clinical outcome of renal transplant recipients: a long-term prospective cohort study. Nephrol Dial Transplant. 2016;31:1168-74.

22. Patel RK, Mark PB, Johnston $\mathrm{N}$ et al. Renal transplantation is not associated with regression of left ventricular hypertrophy: a magnetic resonance study. Clin J Am Soc Nephrol. 2008;3:1807-11.

23. Hewing B, Dehn AM, Staeck $O$ et al. Left Ventricular Structure and Function After Successful Kidney Transplantation. Kidney Blood Press Res. 2016;41:701-9.

24. Rumman RK, Ramroop R, Chanchlani R, Ghany M, Hebert D, Harvey EA, Parekh RS, Mertens L, Grattan M. Longitudinal assessment of myocardial function in childhood chronic kidney disease, during dialysis, and following kidney transplantation. Pediatr Nephrol. 2017;32:1401-10. 
25. Gong IY, Al-Amro B, Prasad GVR et al. Cardiovascular magnetic resonance left ventricular strain in end-stage renal disease patients after kidney transplantation. 2018;20:83.

26. Foley RN Curtis BM,Randell EW, Parfrey PS Left ventricular hypertrophy in new hemodialysis patients without symptomatic cardiac disease. Clin J Am Soc Nephrol. 2010;5:805-13.

27. Meier-Kriesche HU, Baliga R, Kaplan B. Decreased renal function is a strong risk factor for cardiovascular death after renal transplantation. Transplantation. 2003;75:1291-95.

28. Jay CL, Dean PG, Helmick RA, Stegall MD Reassessing Preemptive Kidney Transplantation in the United States: Are We Making Progress? Transplantation. 2016;100:1120-27.

29. Faul C, Amaral AP, Oskouei B et al. FGF23 induces left ventricular hypertrophy. J Clin Invest. 2011;121:4393-408.

\section{Tables}

Table 1. The demographic and clinical characteristics.

\begin{tabular}{|l|l|l|l|}
\hline & Group 1 (n=31) & Group 2 (n=48) & P \\
\hline Age, years, median (IQR) & $64(54-69)$ & $62.5(53.5-68.5)$ & 0.84 \\
\hline Gender female & $14(45.2 \%)$ & $28(58.5 \%)$ & 0.25 \\
\hline BMI, m ${ }^{2}$ kg mean SD & $27.12(3.90)$ & $25.48(4.39)$ & 0.09 \\
\hline HD, months, median (IQR) & $24(12-37)$ & $15(7-24)$ & 0.014 \\
\hline HD>27,5 months & $15(48.4 \%)$ & $9(18.8 \%)$ & 0.005 \\
\hline KTx, months, median (IQR) & $139(56-179)$ & $116(48.5-147.5)$ & 0.22 \\
\hline No HD before KTx & $0(0 \%)$ & $10(20.8 \%)$ & \\
\hline DM & $9(29.0 \%)$ & $12(25.0 \%)$ & 0.75 \\
\hline HTN & $27(87.1 \%)$ & $45(93.7 \%)$ & 0.76 \\
\hline SBP & $134.51(12.06)$ & $139.06(13.89)$ & 0,14 \\
\hline DSP & $79.19(9.4)$ & $80.62(8.09)$ & 0.47 \\
\hline Immunosupresive regimen based on mTORi & $12(38.7 \%)$ & $20(41.7 \%)$ & 0.79 \\
\hline
\end{tabular}

Data are shown as number (percentage), mean and standard deviation for the normal distribution, median and interquartile range for non- normally distribution. Group 1 represent patients with GLS $\geq-18 \%$, whereas Group 2 - GLS $<-18 \%$. 
BMI - body mass index, DM - diabetes mellitus, HD - haemodialysis, HTN - hypertension, KTx - kidney transplantation, mTORi- mammalian target of rapamycin inhibitors, SBP systolic blood pressure, DBP diastolic blood pressure

SD- standard deviation

IQR interquartile range

Table 2. The laboratory results.

\begin{tabular}{|l|l|l|l|}
\hline & Group 1 (n=31) & Group 2 (n=48) & P \\
\hline Total cholesterol, mg\%, median (IQR) & $211(168-244)$ & $224(182-242)$ & 0.73 \\
\hline HDL, mg\%, median (IQR) & $55(44-64)$ & $58(46-70)$ & 0.11 \\
\hline LDL, mg\%, mean (SD) & $129.6(41.4)$ & $127.6(40.9)$ & 0.83 \\
\hline Triglycerides, mg\%, median (IQR) & $153(108-208)$ & $154.5(113-205)$ & 0.87 \\
\hline Serum creatinine, mg\% median (IQR) & $1.50(1.32-1.84)$ & $1.49(1.18-1.71)$ & 0.40 \\
\hline Mean eGFR, mL/min/1.73 m² mean (SD) & $42.94(15.77)$ & $45.52(16.36)$ & 0.49 \\
\hline Uric Acid, mg\%, mean (SD) & $6.92(1.42)$ & $6.91(1.62)$ & 0.98 \\
\hline
\end{tabular}

Data are shown as number (percentage), mean and standard deviation for the normal distribution, median and interquartile range for non- normally distribution. Group 1 represent patients with GLS $\geq-18 \%$, whereas Group 2 - GLS $<-18 \%$.

eGFR - estimated glomerular filtration rate, HDL - high-density lipoprotein cholesterol, LDL - low-density lipoprotein cholesterol.

SD- standard deviation

IQR interquartile range

Table 3. The echocardiographic parameters. 


\begin{tabular}{|c|c|c|c|}
\hline & Group $1(n=31)$ & Group $2(n=48)$ & $\mathrm{P}$ \\
\hline LVDd, mm, mean (SD) & $51.0(5.5)$ & $48.5(5.7)$ & 0.052 \\
\hline IVDd, mm, median (IQR) & $14(12-15)$ & $13.0(12-14)$ & 0.09 \\
\hline PWd, mm, median (IQR) & 11. $(10-12)$ & $11(10-11)$ & 0.29 \\
\hline LAVI, ml/m², median (IQR) & $34.0(32-46)$ & $35.0(31-37.5)$ & 0.14 \\
\hline EF, \%,median (IQR) & $58.0(55.9-61.2)$ & $60.8(59.4-65.0)$ & 0.015 \\
\hline LVMI in women, $\mathrm{g} / \mathrm{m} 2$ median (IQR) & 113.8(97.4-140.3) & $115.4(100-138.8)$ & 0.86 \\
\hline LVMI in men $\mathrm{g} / \mathrm{m} 2$, mean (SD) & $145.4(31.0)$ & $132.6(31.1)$ & 0.22 \\
\hline Without LVH & $5(16.1 \%)$ & $9(18.8 \%)$ & 0.77 \\
\hline Severe LVH & $14(45.2 \%)$ & $17(35.4)$ & 0.39 \\
\hline RWT, median (IQR) & $0.43(0.40-48)$ & $0.44(0.41-0.49)$ & 0.51 \\
\hline Excentric LVH & $6(19.4 \%)$ & $7(14.6 \%)$ & 0.58 \\
\hline Concentric LVH & $15(48.4 \%)$ & $23(47.9 \%)$ & 0.97 \\
\hline $\mathrm{E}, \mathrm{cm} / \mathrm{s}$ mean $(\mathrm{SD})$ & $67.78(21.9)$ & $66.2(14.7)$ & 0.71 \\
\hline $\mathrm{A}, \mathrm{cm} / \mathrm{s}$ mean $(\mathrm{SD})$ & $79.8(20.4)$ & $84.2(16.8)$ & 0.30 \\
\hline DT, ms, median (IQR) & $237(186-300)$ & 218.5(188.5-247.0) & 0.40 \\
\hline e lateral, $\mathrm{cm} / \mathrm{s}$, median (IQR) & $7(6-9) 6$ & $8.0(6-9)$ & 0.41 \\
\hline a lateral, $\mathrm{cm} / \mathrm{s}$, median (IQR) & $9(7-11)$ & $10(9-12)$ & 0.037 \\
\hline e septal, $\mathrm{cm} / \mathrm{s}$, median (IQR) & $6(5-7)$ & 6. (5-8) & 0.74 \\
\hline a septal, $\mathrm{cm} / \mathrm{s}$, median (IQR) & $9(8-11)$ & $10(8-11)$ & 0.85 \\
\hline $\mathrm{E} / \mathrm{e}$ average $>10$ & $23(74.2 \%)$ & $37(77.1 \%)$ & 0.77 \\
\hline $\mathrm{E} / \mathrm{e}$ average $<10$ & $11(35.5 \%)$ & $19(39.6 \%)$ & 0.71 \\
\hline E/e average $>14$ & $3(9.7 \%)$ & $2.4(2 \%)$ & 0.33 \\
\hline GLS average, $\%$, mean (SD) & $-16.3(1.9)$ & $-21.3(2.1)$ & $<0.005$ \\
\hline
\end{tabular}

Data are shown as number (percentage), mean and standard deviation for the normal distribution, median and interquartile range for non- normally distribution. Group 1 represent patients with GLS $\geq-18 \%$, whereas Group 2 - GLS $<-18 \%$. 
GLS - global longitudinal strain, LVH - left ventricular hypertrophy, LVDd - left ventricle end-diastolic diameter, LVDs - left ventricle end-systolic diameter, EF - ejection fraction, LAd - left atrium diameter, LVM - left ventricle mass, LWMI - indexed left ventricle mass, E early mitral flow, A - atrial mitral flow, e - early mitral annulus flow, a - atrial annulus mitral flow, DT - deceleration time.

SD- standard deviation

IQR interquartile range

\section{Figures}

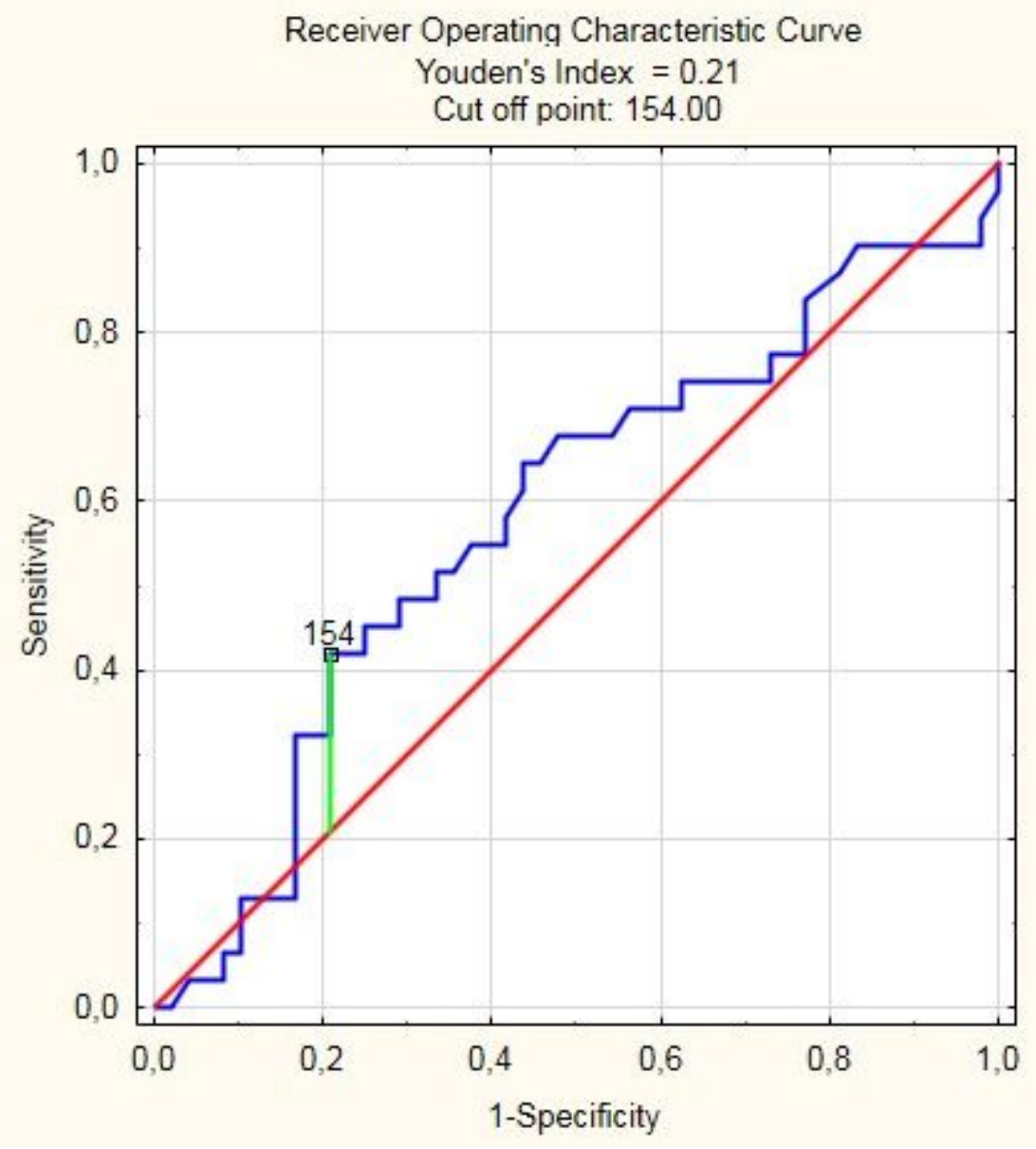

Figure 1

ROC curve determining the cut-off point for hemodialysis time associated with impaired of GLS $\geq-18 \%$ GLS global longitudinal strain HD- hemodialysis 


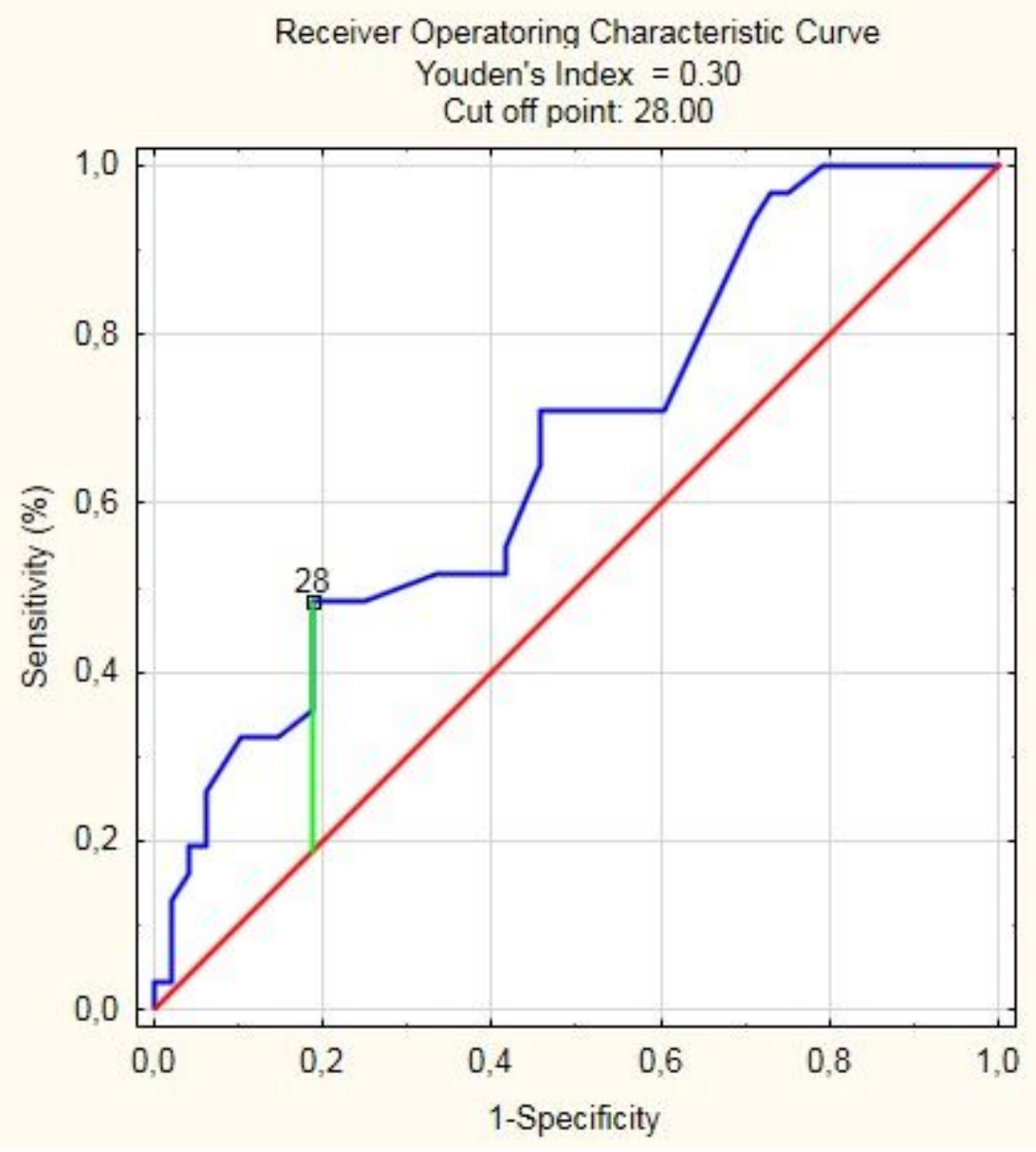

Figure 2

ROC curve determining the cut-off point for time after kidney transplantation associated with impaired of GLS $\geq-18 \%$ GLS global longitudinal strain 